\title{
Investigation of a lattice gas model for surface gravity waves
}

\author{
J. M. Buick and C. A. Greated \\ Department of Physics and Astronomy, The University of Edinburgh, The Kings Buildings, Mayfield Road, \\ Edinburgh, EH9 3JZ, United Kingdom \\ W. J. Easson \\ Department of Mechanical Engineering, The University of Edinburgh, The Kings Buildings, Mayfield Road, \\ Edinburgh, EH9 3JZ, United Kingdom
}

(Received 10 December 1996; accepted 9 May 1997)

\begin{abstract}
A recently proposed lattice gas model for simulating surface waves at a free boundary is described in detail. Simulated waves are compared to linear theory and are seen to compare well, provided an additional parameter $\epsilon$ is introduced. This parameter, $\epsilon$, is investigated and found to be required due to the density gradient produced across the fluid by the gravitational interaction. Its value and the values of the other model parameters are found for a range of gravitational strengths. (C) 1997 American Institute of Physics. [S1070-6631(97)01009-X]
\end{abstract}

\section{INTRODUCTION}

The study and numerical simulation of surface gravity waves has many important applications in oceanography, the safety of ships, the design of oil platforms, the processes of coastal erosion, and the generation of wave power. There are a number of standard numerical techniques such as finite difference, finite element, and boundary integration which can be applied to simulating flows with a free surface, see for example Refs. 1 and 2. When applying these traditional numerical methods to surface wave simulations, major problems may arise in satisfying the dynamic conditions at the free surface, particularly when breaking waves are considered, when the surface elevation may become a multi-valued function of the horizontal coordinate(s). This limits their application to the early stages of wave breaking. ${ }^{3,4}$ The lattice gas model is a more recent numerical tool for the study of general fluid motion and has only very recently been applied to the simulation of surface waves. ${ }^{5}$ Although less developed than the traditional numerical techniques, lattice gas simulations have features which make them appealing in certain situations. Any boundary, ranging from a flat plate to a complex, random surface can be implemented equally efficiently; this has been utilized in the study of flow through porous media, ${ }^{6}$ an application which is of great importance in oil exploration. ${ }^{7}$ The implementation of the model is always performed on a regular grid which, in contrast to the irregular grids used in some traditional simulations, makes the lattice gas model ideally suited to benefit from the high performance of massively parallel computers. Another advantage of the lattice gas model which is of particular relevance to surface wave modeling is that the surface appears in the model in a "natural" way. The position of the surface can be found at any time, but there is no algorithmic requirement to track it; the simulation is treated in the same way at the interface as it is within the liquid and within the gas. This suggests that the lattice gas model could be useful in the study of wave phenomena, including breaking, although it is first necessary to fully test the model and determine the effect of the model parameters, which is the purpose of this paper.

It has been shown ${ }^{5}$ that surface waves can be produced using an $\mathrm{FHP}^{8}$ model with long-range interactions ${ }^{9}$ and a gravitational interaction incorporated. Waves simulated in this way give good agreement with the predictions of linear wave theory, although there is some discrepancy between the actual wavelength-to-depth ratio used in the simulation and the apparent wavelength-to-depth ratio observed in the model. The viscosity of the fluid, $\nu$, and an important scaling parameter, $\gamma$, were discussed in Ref. 5, but their numerical values were not considered. The "strength" of the gravitational interaction was measured in terms of a parameter $S_{g}$. This is a measure of the ratio of the number of actual implementations of the interaction to the number of possible implementations, a parameter which is useful in the formulation of the computer algorithm but which is not associated with the physical strength of the interaction. In this paper we present the results of numerous simulations performed for different values of the model parameters. The effect of the gravitational interaction is considered and the implementation parameter $S_{g}$ is related to the acceleration due to gravity $g$. We look at the effect of the density gradient produced across the fluid by the gravitational interactions and show that this can be accounted for by rescaling the depth by a new parameter, $\epsilon$. This new parameter is seen to depend on the strength of the gravitational interactions and its value is found for relevant gravitational strengths. The values of the viscosity $\nu$ and the scaling parameter $\gamma$ are considered and found experimentally from the simulations.

\section{THE MODEL}

We now review the lattice gas model ${ }^{5}$ used in the simulations. It is based on the $\mathrm{FHP}^{8}$ model of Frisch, Hasslacher, and Pomeau which was first introduced in 1986 and has since been used in a variety of fluid simulations ranging from single fluid simulations such as flow round a plate ${ }^{10}$ and flow in a pipe ${ }^{11}$ to multiple fluid simulations. ${ }^{12-14}$ Their technique has been altered in a number of ways ${ }^{14,15,9,16}$ to allow it to model different phenomena. Here we use the long-range interaction first proposed by Appart and Zaleski ${ }^{9,16}$ and a gravitational interaction. These models and their basic properties are described below. 


\section{A. Definition of a lattice gas model}

Lattice gas models belong to the class of cellular automata and are used for simulating fluid systems. A cellular automata consists of a lattice whose sites, the intersection points of the lattice, can take on a finite number of states. The automaton evolves in discrete time steps; the state of each site at any time is determined by the state of a set of neighboring sites at the previous time step. Typically the number of neighbor sites required to update a site is small. In the FHP model a hexagonal lattice is used and each site is updated from the state of the site at the previous time step and the state of the six nearest neighbor sites. The introduction of the long-range interaction requires that the state of sites three lattice steps away is known for each update; the gravitational interactions require only the previous state of the site be known.

\section{B. The FHP model}

A hexagonal grid is used for the FHP model. Each of the sites are connected to their six nearest neighbors by the links $\mathbf{e}_{i}=\sin (\pi i / 3-\pi / 6) \mathbf{i}+\cos (\pi i / 3-\pi / 6) \mathbf{j}, \quad i=1, \quad 2, \ldots, 6$. The site is considered to be the link $\mathbf{e}_{0}$. Fluid "particles" are allowed to travel with unit speed along the links $\mathbf{e}_{1}-\mathbf{e}_{6}$ or remain at rest in link $\mathbf{e}_{0}$ and an exclusion principle is applied allowing only one particle to travel in each direction along a link. Each of the fluid "particles" is considered to have unit mass. The model evolves from time $t$ to time $t+1$ in two steps: the streaming step $\mathscr{S}$ and the collision step $\mathscr{C}$. In the streaming step the particles on links $\mathbf{e}_{1}-\mathbf{e}_{6}$ move from their original site to one of the six neighboring sites, any particle on link $\mathbf{e}_{0}$ remains at rest at the site. In the collision step any particles newly arriving at a site and any rest particle already present at the site are allowed to collide in such a way that the mass (particle number) and the momentum are conserved at each site on the grid. Here we use the FHP-III collision rules; that is all possible collisions which conserve both mass and momentum are allowed. Various subsets of these rules make up the FHP-I and FHP-II collision rules. A set of collision rules on a hexagonal lattice is shown in Fig. 1, where the small filled circles represent the lattice site and the large empty circles represent a rest particle at the site. The lefthand column represents the possible incoming configurations or in-states. The right-hand column represents the outgoing configurations or out-states. When there are two different possible outcomes one of the choices is picked at random. The full set of collisions can be obtained by combining the collisions of Fig. 1 with the collisions found by rotating the particles through multiples of $\pi / 3$ and by considering the duals of all the collision formed by swapping the full and empty links. It can clearly be seen that all the individual rules conserve both mass and momentum at each site. There are 76 possible collisions: 15 two-particle collisions of type (a), (c), and (d); 23 three-particle collisions of type (b), (e), and (f); 23 four-particle collisions and 15 five-particle collisions. The four- and five-particle collisions are the dual of the three and two particle collisions respectively. The dual of a collision is found by adding particles to the empty links and removing particles from the links which were originally a)
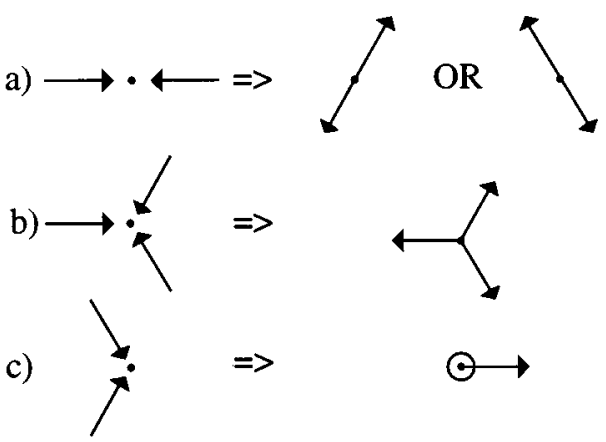

d)
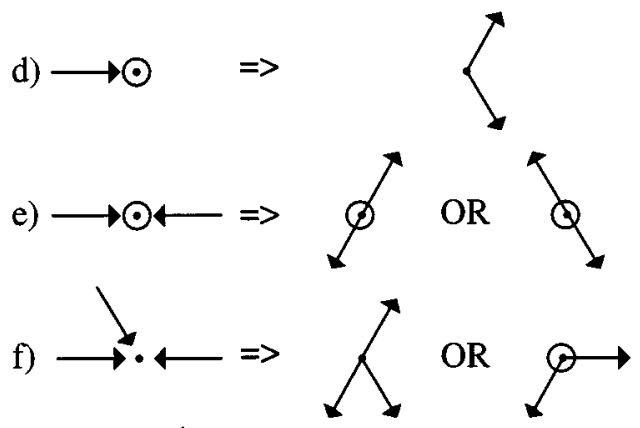

g)
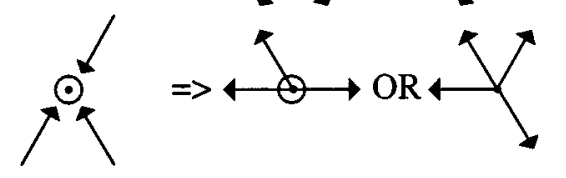

FIG. 1. Collision rules for the FHP model. The sites are represented by the small filled circles and rest particles are represented by the large empty circles.

filled: example ( $\mathrm{g}$ ) is the dual of example (f). There are a total of 18 collisions of type (f) which can be considered to be collisions of type (a) or (c) with a spectator particle (a particle which does not take part in the collision and which continues traveling in a straight line). There are two possible outcomes in example (f) because it can be thought of as either example (a) with a spectator particle traveling on link $\mathbf{e}_{6}$ or as example (c) rotated through $2 \pi / 3$ with a spectator particle traveling on link $\mathbf{e}_{5}$. One restriction with the FHP-III model is that collisions (b) and (e) are not interchangeable; that is, the (e) in-state cannot collide to give the (b) out-state even though this conserves mass and momentum. This restriction has no effect on the properties of the model, but simplifies its implementation since it restricts the maximum possible number of out-states for each in-state to two. Since the particles travel at unit speed along the lattice, or remain at rest, it is possible to completely discretize the model. Only the state of each site at the previous time step is required to calculate the state of each site at the next time step. In practice, this was modeled using a 7-bit integer (between 0 and 127) to represent the state $s$ of each site, each bit $s_{i}, i=1,7$ being 1 if there is a particle on link $\mathbf{e}_{i+1}$ and 0 otherwise. The streaming operation is performed by shifting the grid in each of the six link directions and using bit arithmetic. The collisions are performed using a lookup table which takes the in-state as input and outputs the out-state. A solid boundary can be inserted in the model. Any particle arriving at a boundary site is either reflected back along the link it approached on (no-slip boundary) or it is reflected so that the 
momentum perpendicular to the boundary is reversed and the momentum parallel to the wall is conserved (free-slip boundary). Thus a particle approaching a horizontal boundary along link $\mathbf{e}_{4}$ will move off along link $\mathbf{e}_{1}$ if it is a no-slip boundary and along link $\mathbf{e}_{6}$ if it is a free-slip boundary. Provided the collision rules satisfy the conservation of mass and momentum, that is,

$$
\sum_{i} n_{i}\left(t+1, \mathbf{r}+\mathbf{e}_{i}\right)=\sum_{i} n_{i}(t, \mathbf{r})
$$

and

$$
\sum_{i} \mathbf{e}_{i} n_{i}\left(t+1, \mathbf{r}+\mathbf{e}_{i}\right)=\sum_{i} \mathbf{e}_{i} n_{i}(t, \mathbf{r}),
$$

at each site, it can be shown ${ }^{17,18}$ that

$$
\partial_{t} \rho+\nabla \cdot \rho \mathbf{u}=0
$$

and

$$
\partial_{t} \mathbf{u}+\gamma(d) \mathbf{u} \cdot \nabla \mathbf{u}=-\nabla P+\nu \nabla^{2} \mathbf{u},
$$

where $\rho$ is the density, $P$ is the pressure, $d$ is the density per link, $d=\rho / 7, \nu$ is the viscosity which is found to be a function of the density, and $\gamma(d)$ is a density-dependent function. For the FHP-III model

$$
\gamma(d)=\frac{7(1-2 d)}{12(1-d)}
$$

and

$$
\nu=\frac{1}{28} \frac{1}{d(1-d)} \frac{1}{1-8 d(1-d) / 7}-\frac{1}{8} .
$$

It is possible to rescale certain variables in Eq. (4) in order to produce the Navier-Stokes equation. Following Wolfram ${ }^{18}$ we can define $\widetilde{\mathbf{u}}=\gamma(d) \mathbf{u}$ and $\widetilde{P}=\gamma(d) P$ so that Eq. (4) gives the Navier-Stokes equation in the rescaled variables. The Reynolds number is given by

$$
\mathrm{Re}=\frac{\widetilde{U} L}{\nu(d)}=\frac{U L \gamma(d)}{\nu(d)} .
$$

The form of the viscosity, $\nu(d)$, depends on the grid used and also on the allowed collisions while the scaling factor, $\gamma(d)$, which is required due to the non-Galilean invariance of the model at the microscopic level, ${ }^{17}$ depends on the grid which is being used and not on the set of allowed collisions. All the results presented here will be unscaled measurements. Where required the ' $\gamma$ ' factor will be introduced into the equation of motion.

\section{Long-range interactions rules}

The long-range interaction rules were first introduced by Appert and Zaleski. ${ }^{9}$ The interaction was further developed in Ref. 16. The interactions used here are effectively the same as these interactions, however the implementation varies slightly. ${ }^{5}$ Figure 2 shows the basic interactions acting along the $\mathbf{e}_{2}$ direction on two sites a distance $R$ apart. The interaction can take place only if there are particles traveling in both directions indicated by the solid arrows and if there are no particles traveling in the direction of either of the
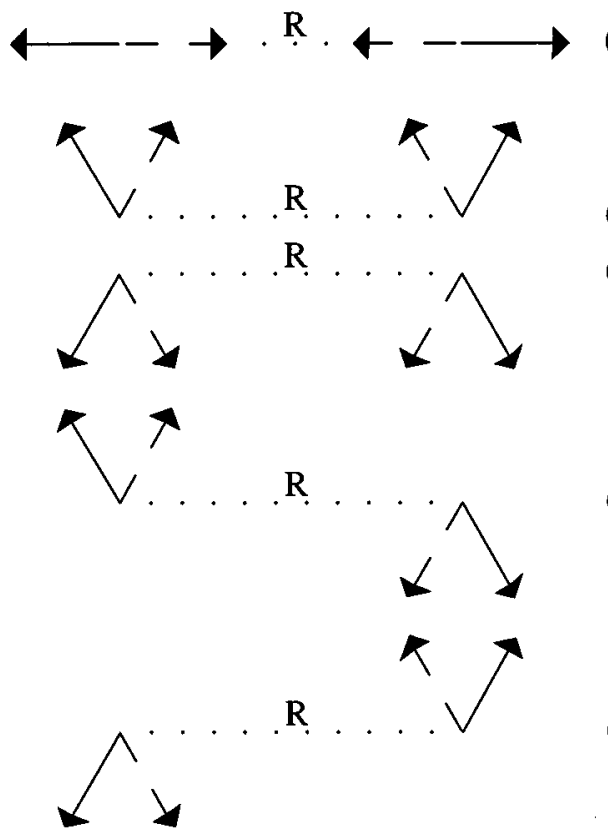

(d)

FIG. 2. The basic long-range interactions acting in the direction of $\mathbf{e}_{2}$. Particles at sites $R$ lattice units apart are flipped from the solid link directions to the dashed directions provided there is initially a particle in both the solid links and no particles in either of the dashed links.

dashed arrows. If this is the case, the particles are flipped from the solid link to the dashed link. These five basic interactions are also applied along the $\mathbf{e}_{1}$ and $\mathbf{e}_{3}$ directions.

Let $\mathscr{J}_{i}^{(x)}, \forall x \in\{a, b, c, d, e\}, i \in\{1,2,3\}$ be the operator which implements interaction $x$, from Fig. 2, along direction $\mathbf{e}_{i}$. If the interaction cannot take place the operator $\mathscr{J}_{i}^{(x)}$ defaults to the identity operator, $\mathscr{T}$, leaving the particles unchanged. If we define the operators

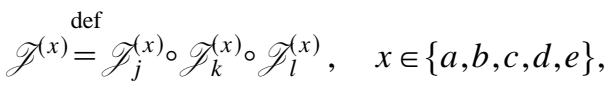

where $j, k, l$ are a random ordering or $1,2,3$, then we can define an overall interaction operator

$$
\mathscr{J}^{\operatorname{def}}=\mathscr{J}^{(a)} \circ \mathscr{J}^{(b, c)} \circ \mathscr{J}^{(d, e)},
$$

where

$$
\mathscr{J}^{(b, c)}= \begin{cases}\mathscr{J}^{(b)} & \text { if only } b \text {-flip is possible } \\ \mathscr{J}^{(c)} & \text { if only } c \text {-flip is possible, } \\ \mathscr{J}^{(b)} \circ \mathscr{J}^{(c)} & \text { if both flips are possible }\end{cases}
$$

and $\mathscr{J}^{(d, e)}$ is defined similarly. The combining of interactions $\mathscr{J}^{(d)}$ and $\mathscr{J}^{(e)}$ is required to prevent unwanted rotations being produced in the flow. Interactions $\mathscr{J}^{(b)}$ and $\mathscr{J}^{(c)}$ are combined for simplicity. The long-range interactions have the effect of separating the fluid into two distinct phases. ${ }^{9,16}$ The dense phase has a density of approximately 4.0 particles per site and the rare phase has a density of approximately 0.1 particles per site when $R=3$. A definite interface is formed between the two fluids. If a larger value of $R$ is used, there is a larger difference between the densities of the two fluids, 


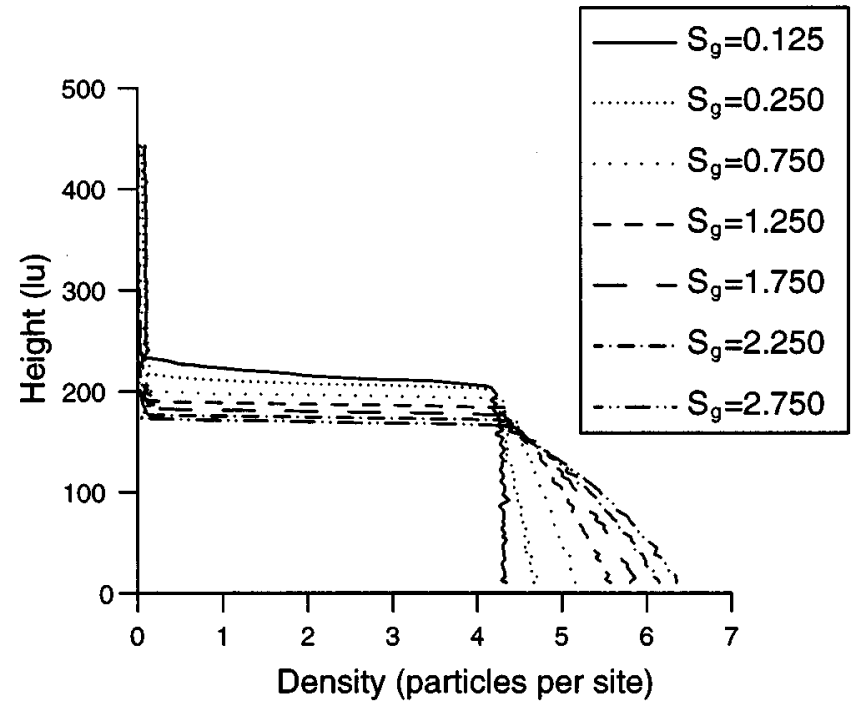

FIG. 3. Height plotted against density for different values of $S_{g}$. The graphs have been smoothed slightly to enhance their clarity.

however, using too large a value, greater than 7 , causes internal structure to appear within the dense fluid. $R=3$ is used in all the results presented here.

\section{Gravitational interactions}

Body forces can be introduced into the model by flipping particles so as to change the momentum at a site. Gravitational interactions can be simulated on a hexagonal grid by flipping particles, after the collision, from link $\mathbf{e}_{1}$ to link $\mathbf{e}_{3}$ and from $\mathbf{e}_{6}$ to $\mathbf{e}_{4}$, provided there is not already a particle on the destination link. The strength of the interaction will depend on the number of particles flipped in a single time step. This is done by introducing a gravitational operator $\mathscr{G}=\mathscr{G}_{1}+\mathscr{G}_{2}$, where $\mathscr{G}_{1}$ flips a particle from $\mathbf{e}_{1}$ to $\mathbf{e}_{3}$ with probability $S_{g} / 100$ and $\mathscr{G}_{2}$ flips a particle from $\mathbf{e}_{6}$ to $\mathbf{e}_{4}$ with probability $S_{g} / 100$ and both $\mathscr{G}_{1}$ and $\mathscr{G}_{2}$ default to $\mathscr{T}$, the identity operator, if there is no particle in the original link or a particle is already in the destination link. Thus both $\mathscr{G}_{1}$ and $\mathscr{G}_{2}$ have the effect of introducing a momentum change in the negative horizontal direction $S_{g} \%$ of the time that such an interaction is possible.

Introducing gravity as well as the long-range interaction produces the same separation, but now the dense phase is situated at the bottom of the grid and the rare phase at the top. An approximately horizontal interface separates the two fluids. This can be seen in Fig. 3 for different values of $S_{g}$ where the curves have been smoothed slightly for clarity. We see that the strength of the gravitational interaction has little effect on the fluid densities at the interface. The strength of the interaction does, however, produce a density gradient across both fluids, the gradient being larger for larger values of $S_{g}$. For $S_{g} \leqslant 0.75$ the gradient is approximately linear.

\section{E. Model implementation and wave simulations}

The model is allowed to evolve under the action of all the processes described above. Thus the system evolves according to the evolution operator

$$
\mathscr{E}=\mathscr{S} \circ \mathscr{J} \circ \mathscr{G} \circ \mathscr{C} \text {. }
$$

When this operator is applied to a grid with an initially random density profile and zero average velocity with a solid boundary at the top and bottom and continuous boundary conditions at the other two edges, the fluid separates to give a density profile as shown in Fig. 3, and the average velocity once it has reached equilibrium will still be zero. The height at which the interface occurs will depend only on the average density of the initial setup. Once the fluid has evolved to this steady state a standing wave at an extreme of its oscillation can be inserted at the interface. ${ }^{5}$ This is done by superimposing a sinusoidal interface of the required wavelength and amplitude over the horizontal surface. The sites which were previously above the horizontal interface but are now below the sinusoidal surface are filled with particles so they have an average density of 4.0 particles per site and zero average velocity. The sites which were initially below the horizontal interface but are now above the sinusoidal surface are filled with average density 0.1 and zero average velocity. Any amplitude of wave can be inserted but here we restrict ourselves to small amplitudes so that the wave falls into the linear range. The wavelength used is always the horizontal size of the grid.

\section{MAKING MEASUREMENTS FROM THE SIMULATIONS AND THEIR ERRORS}

Measurements made from the lattice are in terms of lattice units (lu). The unit of length is the lattice spacing and the unit of time is the time step. Velocities are measured in lattice lengths per time step and densities in particles per site. The microscopic density $\rho$ and velocity $u$ at each site are defined by

$$
\rho(t, \mathbf{r})=\sum_{i} n_{i}(t, \mathbf{r})
$$

and

$$
\rho \mathbf{u}(t, \mathbf{r})=\sum_{i}^{\text {def }} \mathbf{e}_{i} n_{i}(t, \mathbf{r}) .
$$

Macroscopic quantities are found by averaging the microscopic quantities over a number of sites. Three types of averaging are possible: spatial averaging, temporal averaging, and ensemble averaging. Spatial averaging can always be used efficiently since the spatial scale of the simulated flow must always be large compared with the lattice spacing if the model is to produce meaningful results. Temporal averages can also be used effectively either when a steady state flow is being simulated or for a flow which varies on a time scale significantly larger than the averaging time being used. Ensemble averaging can always be used by running a simulation a number of times starting from the same initial conditions but using a different random seed during the 
simulation. All measurements of velocity and density taken here are spatially averaged over at least 256 sites and often significantly more. In a small number of stated occasions ensemble averaging was also used to reduce the noise. The error in the density and velocity measurements can be shown to $\mathrm{be}^{19}$

$$
\Delta \rho=1.128 \sqrt{\frac{d(1-d)}{7 S}}
$$

and

$$
\Delta u=C(\rho) S^{-1 / 2},
$$

where $S$ is the number of sites averaged over and $C(\rho)$ is no larger then 0.28 for the densities used in our simulations.

During the simulation the height of the center of the wave is recorded every 40 time steps. This is done by considering a column 16 sites wide about the center of the wave. The wavelength used in all cases is much larger than 16 units. The density is then measured in each row of the column and the height of the surface found; this is taken to be the height at which the density drops below two particles per site. The averages of these 16 results are then fitted to a curve of the form

$$
A e^{-\alpha t} \cos \left(\frac{2 \pi t}{\tau}+\psi\right)+h,
$$

where $A$ is the initial amplitude, $\alpha$ is the damping constant, $\tau$ is the period, $h$ is the mean water depth, and $\psi$ is a phase difference in the range $-\pi<\psi<\pi$ introduced to account for any inaccuracies in initializing the wave and any small initial time before the wave starts to oscillate. We expect $\psi$ to be small if the wave has been initialized correctly; typically we found $|\psi| \ll \pi$.

There will be many sources of error which contribute to the total error in the fitted parameters. In particular the amount of time over which data are acquired will be important in determining the size of the error. In order to asses this effect a wave was initialized on a 2048 by 128 grid with $h$ $=57 \mathrm{lu}, \lambda=2048 \mathrm{lu}$, and $A=22 \mathrm{lu}$. The wave was allowed to evolve using free-slip boundary conditions for 20000 time steps and the height of the wave at its center was recorded every 40 time steps. These results were then analyzed using the curve-fitting routine 20 times, each time the results for the first $n$ thousand time steps were considered, $n=1,2, \ldots, 20$. The values of the fitted parameters are shown in Fig. 4, normalized by their final value, found after 20000 time steps, plotted against the number of periods of the wave which were considered in the curve fitting. The period of the wave was calculated from the results for $t$ $=20000$. Note that some of the parameters found from less than one period of results lie outside the range of the graph. These results suggest that, provided more than one period of the wave is analyzed, the parameters are found to vary by less than $3 \%$ from their mean value with the exception of $\alpha$ which varies by no more than $10 \%$. If more than two periods are analyzed the variation in $\alpha$ is within 3\% of its mean value as are the other parameters.

It is also important to be aware of the repeatability of the experiments. To investigate this, a wave was set up on a

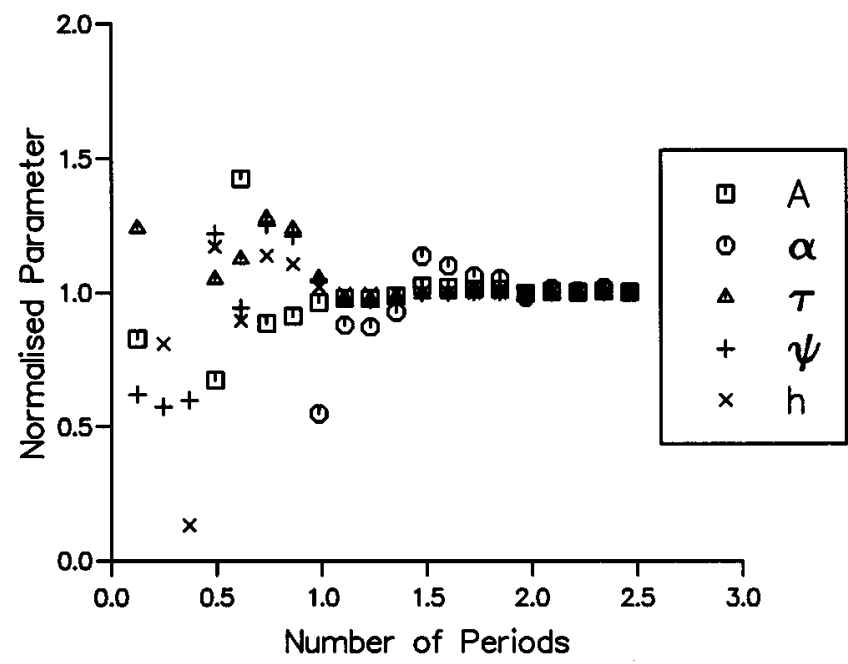

FIG. 4. The normalized fitted parameters plotted against the number of wave periods which were considered in the fitting procedure.

2048 by 256 lattice with wavelength 2048 lu, initial amplitude $22 \mathrm{lu}$, and mean water level $191 \mathrm{lu}$. This simulation was run seven times using different random seeds and each run was fitted to a curve. Each simulation was run for 7000 time steps $\sim 1.25$ periods. The percentage standard deviations found in the fitted parameters, $\alpha, \tau$, and $h$, in which we are interested, were found to be 5.7, 3.0, and 0.14, respectively. These results suggest that values of the damping constant, the period, and the mean water depth can be found with reasonable accuracy even if the wave is only allowed to evolve for one and a quarter periods. This allows the results to be taken in a time efficient manner. The error in $\alpha$ will be taken to be $6 \%$ and the error in $\tau$ to be $3 \%$. In every simulation the mean water level, $h$ was always found to be within $0.2 \%$ of the value to which it was initialized, and so this value is used.

\section{EQUATIONS DESCRIBING A LINEAR WAVE}

The following equations (see, for example, Refs. 20 and 21) describe the behavior of linear waves and are used to compare the waves produced here to real waves and to allow us to find value for the model parameters. We are interested here in two-dimensional waves in the $x-y$ plane where the mean water level is at $y=0$ and $h$ is the water depth. The dispersion relation

$$
\omega^{2}=g k \tanh k h
$$

relates the frequency and wave number of waves with a freeslip boundary condition at $y=-h$. The frequency of a wave on a no-slip bed is reduced by a factor

$$
1-(\nu / 2 \omega)^{1 / 2} k /(\sinh 2 k h)
$$

giving a, typically small, decrease in the angular frequency. Damping of waves is caused by internal viscosity in all waves and by bed friction if there is significant wave motion at the bed. The internal viscous effects give a proportional loss of amplitude per unit time of

$2 \nu k^{2}$ 
and bed friction causes a proportional loss of amplitude per unit time of

$$
\left(\frac{\nu \omega}{2}\right)^{1 / 2} \frac{k}{(\sinh 2 k h)} \text {. }
$$

The velocities under a two-dimensional linear standing wave are given by

$$
\begin{aligned}
& v_{x}=\frac{-a \omega \cosh [k(y+h)]}{\sinh (k h)} \sin (k x) \sin (\omega t), \\
& v_{y}=\frac{a \omega \sinh [k(y+h)]}{\sinh (k h)} \cos (k x) \sin (\omega t),
\end{aligned}
$$

where $a$ is the wave amplitude. The velocities at the surface of a wave at $t=\tau / 4, x=\lambda / 4$ are given by

$$
\begin{aligned}
& v_{x}^{(0)}=\frac{a}{\gamma} \sqrt{\frac{g k}{\tanh (k h)},} \\
& v_{y}^{(0)}=\frac{a}{\gamma} \sqrt{g k \tanh (k h)} .
\end{aligned}
$$

Let $h^{*}$ be the depth below which there is only negligible motion due to the surface wave. This is commonly taken to be when

$$
h^{*}=\lambda / 2 \text {, }
$$

where the energy loss due to bed friction is only $2 \%$ of its long wavelength limit. ${ }^{20}$ If $h>h^{*}$, then the viscosity $\nu$ can be related to $\alpha$, first introduced in Eq. (16), using Eq. (19). This gives

$$
\alpha=\beta \nu,
$$

where

$$
\beta=2 k^{2} \text {. }
$$

Energy dissipation due to bottom friction need not be considered here because the velocity at the bottom boundary is negligible. When $h<h^{*}$ and free-slip boundary conditions are used, we can write, assuming that in the shallow water limit the $x$ velocity is independent of $y$ and the $y$ velocity is negligible,

$$
\alpha=\frac{h}{h^{*}} \beta \nu .
$$

When $h<h^{*}$ and no-slip boundary conditions are used the viscosity is found by solving

$$
\alpha=\frac{h}{h^{*}} \beta \nu+\frac{\left(\frac{\nu \omega}{2}\right)^{1 / 2} k}{\sinh 2 k h} .
$$

This comes from Eq. (26) with the attenuation term due to bed friction given in Eq. (20) added.

\section{RESULTS}

Numerous standing wave simulations were performed on the Connection Machine CM-200 at Edinburgh University.

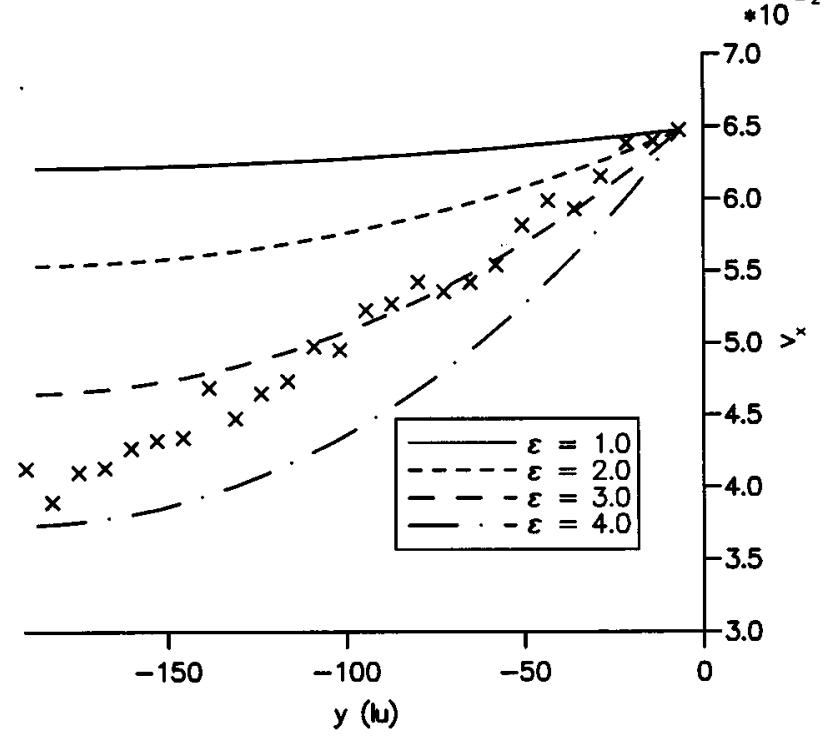

FIG. 5. The variation in the peak $x$ velocity with height for the simulated wave and also the linear theory predictions of the velocity for waves with a depth to wavelength ratio $\epsilon$ times the depth to wavelength ratio of the simulated wave.

The results of these simulations are presented in this section and, through a comparison with theory, the model parameters are studied.

\section{A. The need for a vertical scaling parameter}

We now consider three examples which demonstrate the need to introduce a parameter $\epsilon$ which scales the horizontal displacement, and we observe the effect that such a parameter has on the simulations. All three examples were run with $S_{g}=0.5$.

\section{Velocity variation with depth}

The need to introduce a scaling parameter $\epsilon$ to scale the depth was first observed ${ }^{5}$ when considering the dependence of the wave velocity with depth. This can be seen explicitly here where we consider a wave on a free-slip bed using a 4096 by 256 site grid. A wave with mean water level of 189 lu was set up and allowed to oscillate and the velocity was calculated after a quarter period when it had its maximum value. The velocity was averaged over six ensemble experiments. The $x$ component of the velocity at $x=\lambda / 4$ is shown in Fig. 5 as is the velocity distribution predicted from linear theory for a wave with the same wavelength but with a depth of $\epsilon$ times the simulation depth. That is, when $\epsilon=1$, the velocity was calculated from Eq. (21) while for $\epsilon \neq 1$ the velocities were calculated from

$$
v_{x}=A_{\epsilon} \frac{\cosh [k \epsilon(y+h)]}{\sinh (\epsilon k h)},
$$

where $A_{\epsilon}$ is a normalizing factor which ensures the velocities coincide at the surface $(y=0)$. The velocity within the top $25 \mathrm{lu}$ appears to follow a distribution between the $\epsilon=1.0$ and $\epsilon=2.0$ curves. It then follows the $\epsilon=3.0$ distribution in the next $75 \mathrm{lu}$. For depths greater than 100 lu the measured ve- 


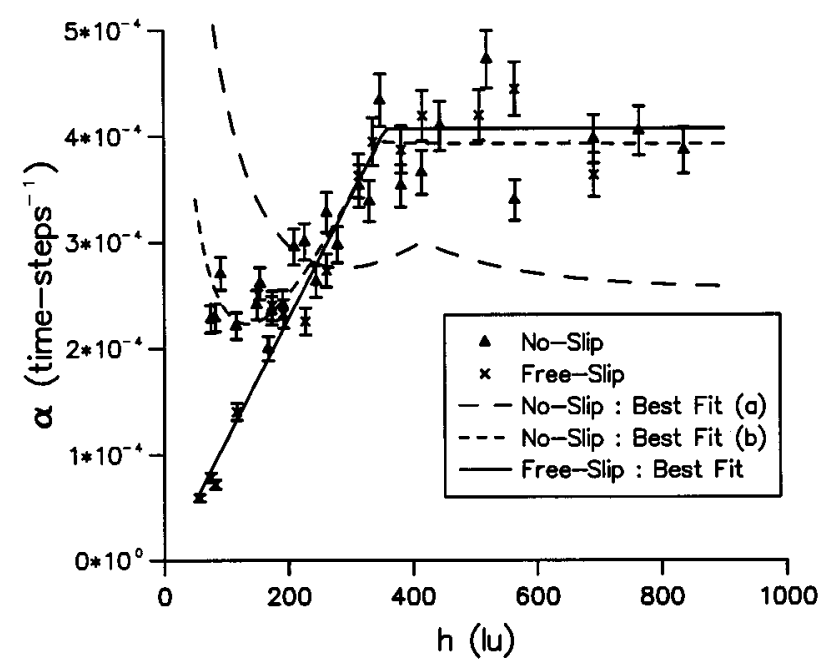

FIG. 6. The variation in the damping constant, $\alpha$, with the mean water level, $h$, for both the no-slip and the free-slip boundary conditions when $S_{g}=0.5$. Also shown are three best-fit curves.

locity distribution is roughly parallel to the $\epsilon=4.0$ curve. Here we see the need to scale the water depth by some parameter $\epsilon$ and that in general $\epsilon$ is itself a function of depth. Despite the variation of $\epsilon$ with depth it can be seen from Fig. 5 that if $\epsilon$ was to take a constant value of about 3 , at all depths a good fit with the data would be obtained. We note also that this approximation is most accurate in the upper section of the wave where the velocities are largest.

\section{The dependence of the damping constant on the mean water level}

Waves of wavelength 2048 lu were initialized for a range of mean water levels. These simulations were then run using no-slip boundary conditions. Some of the simulations were then repeated using free-slip boundary conditions. The values of the damping constant $\alpha$ found from the curve fitting are shown in Fig. 6. Also shown are the following fitted curves:

(a) The no-slip results were fitted to Eq. (24) for $h>h^{*}$ and Eq. (27) for $h \leqslant h^{*}$ to find the best-fit values of $h^{*}$ and $\nu$ : fit (a).

(b) The no-slip results were also fitted to Eq. (24) for $h>h^{*}$ and Eq. (29) for $h \leqslant h^{*}$ to find the best-fit values of $h^{*}, \nu$ and $\epsilon$ : fit (b).

(c) The free-slip results were fitted to Eq. (24) for $h>h^{*}$ and Eq. (26) for $h \leqslant h^{*}$ to find the best-fit values of $h^{*}$ and $\nu$ :

$$
\alpha=\frac{h}{h^{*}} \beta \nu+\frac{\left(\frac{\nu \omega}{2}\right)^{1 / 2} k}{\sinh 2 k \epsilon h} .
$$

The best-fit parameters are shown in Table I along with $e$, the root mean square deviation of the results from the best-fit curve. The fitted curves (b) and (c) show a good agreement with the results with nearly all the points being within two standard deviations of the curves. The fitted curve (a) is
TABLE I. Best-fit parameters for the curves shown in Fig. 6 when $S_{g}$ $=0.5$.

\begin{tabular}{lcccc}
\hline \hline & $h^{*}$ & $\nu$ & $\epsilon$ & $e$ \\
\hline No-slip best fit (a) & 415 & 13.6 & - & $1.121 \times 10^{-4}$ \\
No-slip best fit (b) & 348 & 20.8 & 2.66 & $3.151 \times 10^{-5}$ \\
Free-slip best fit & 357 & 21.6 & - & $2.456 \times 10^{-5}$ \\
\hline \hline
\end{tabular}

clearly not going through the data points. Despite the errors in the measured quantities which will affect the accuracy of the results in Table I, we can clearly see the necessity of introducing $\epsilon$ and the sensitivity of the results to $\epsilon$ when comparing curves (a) and (b). We also see that a good fit can be obtained by assuming that $\epsilon$ is a constant at all depths. A value of 2.66 was found explicitly in fit (b). Since the expression $h / h^{*}$ should properly be written $\epsilon h / \epsilon h^{*}$, we also obtain two implicit values for $\epsilon, 2.89$ and 2.94 when comparing the the values of $h^{*}$ for curves (b) and (c) with the expected value of $\lambda / 2$.

\section{The dependence of the surface velocity on the mean water level}

Velocities under a number of waves in different water depths were measured after a quarter period for a free-slip bed, the period of the wave, as well as the damping constant, having been found previously using the curve-fitting method described above. Figure 7 shows the $x$ components of velocity for six of the waves plotted against height from the bed. Note that the origin has been displaced from the mwl to the bed. This has been done only in Fig. 7 to aid the presentation of the results. From Eq. (22) we expect $v_{x}$ to be constant for $h$ large enough the $\sqrt{\tanh k h} \simeq 1$. This is true to within $5 \%$ for $h>484$ lu. In Fig. 7 the surface velocity appears constant for $h$ as small as $200 \mathrm{lu}$. This is again consistent with introducing a scaling parameter $\epsilon$ into Eq. (22) with a value

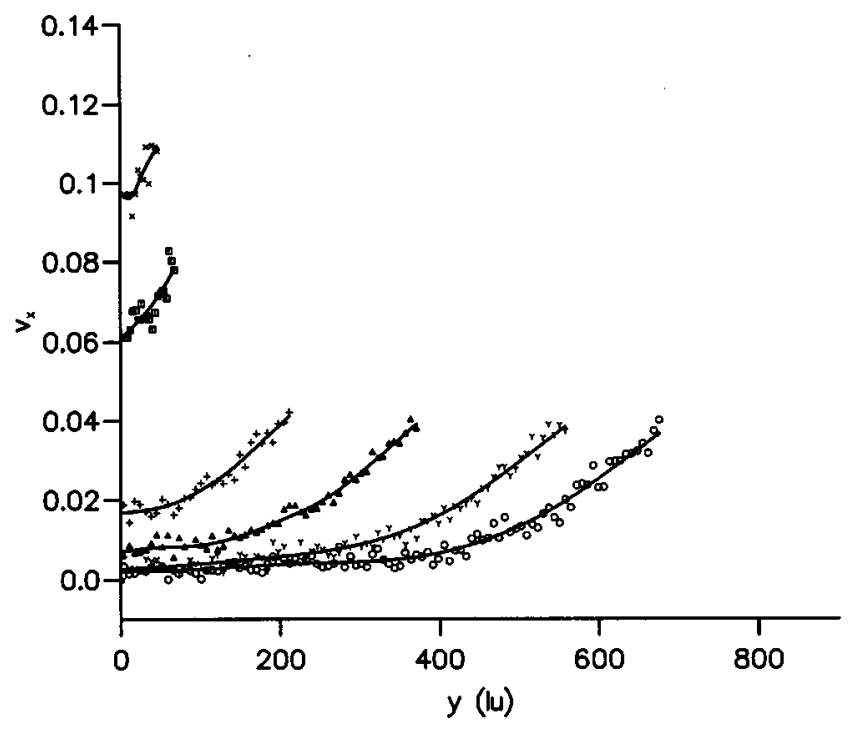

FIG. 7. The variation in $x$ velocity with height for six waves with free-slip boundaries when $S_{g}=0.5$. 


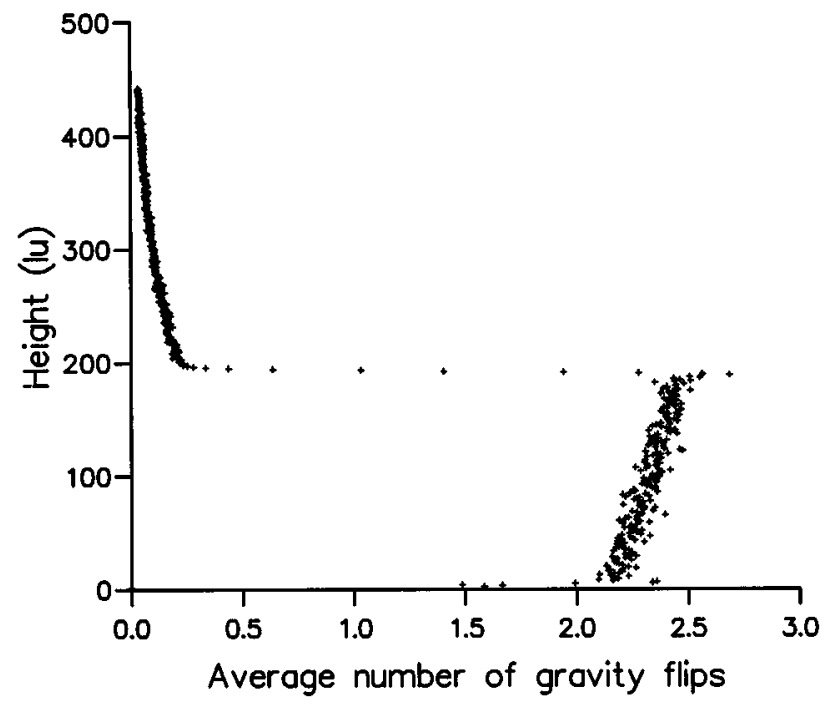

FIG. 8. The average number of gravity flips at different heights above the bottom boundary after 1000 time steps when $S_{g}=0.5$.

$\sim 3$. In the deep water limit we see that $v_{x} \rightarrow 0.042$. For $\alpha=4.1 \times 10^{-4}, \tau=5200, A=20$, and $a=A \exp (-\alpha \tau / 4)$ this gives $\sqrt{g} / \gamma=0.065$, which is consistent with values obtained in the next section.

\section{Summary of results concerning a vertical scaling parameter}

We have seen the need to introduce a scaling parameter $\epsilon$ in the previous examples where the gravitational strength, $S_{g}$ was 0.5 . For this value of $S_{g}$ we can consider $\epsilon$ to be independent of depth and, using this constant value, good agreement is found with linear theory when considering properties of the whole wave and also when considering the velocities of the wave at its surface. When similar experiments are carried out with $S_{g}=0.125$, and hence a negligible density gradient across the fluid, we do not observe the same need to introduce $\epsilon$. We therefore conclude that $\epsilon$ is required to account for the density gradient with $\epsilon=1$ when there is no density gradient and $\epsilon$ increasing as the gradient increases.

\section{B. Determining the values of the model parameters}

We wish to determine the values of the model parameters for different values of $S_{g}$ and to see how the acceleration due to gravity is related to $S_{g}$.

\section{Gravity flips within the fluid}

First we need to consider the number of gravity flips per site per time step, $\mu$. A simulation was run on a 2048 by 512 grid with a flat surface at height $191 \mathrm{lu}$, for 1000 updates using a strength factor $S_{g}=0.5$. The total number of gravity flips was recorded along with the position where they occurred. The average number of flips in each row of the grid was then calculated and is shown in Fig. 8. This shows that the vast majority of the flips take place in the more dense fluid and that within this fluid the flips are fairly evenly distributed with only a gradual variation caused by the density gradient across the fluid. Thus the number of gravity
$* 10^{-3}$

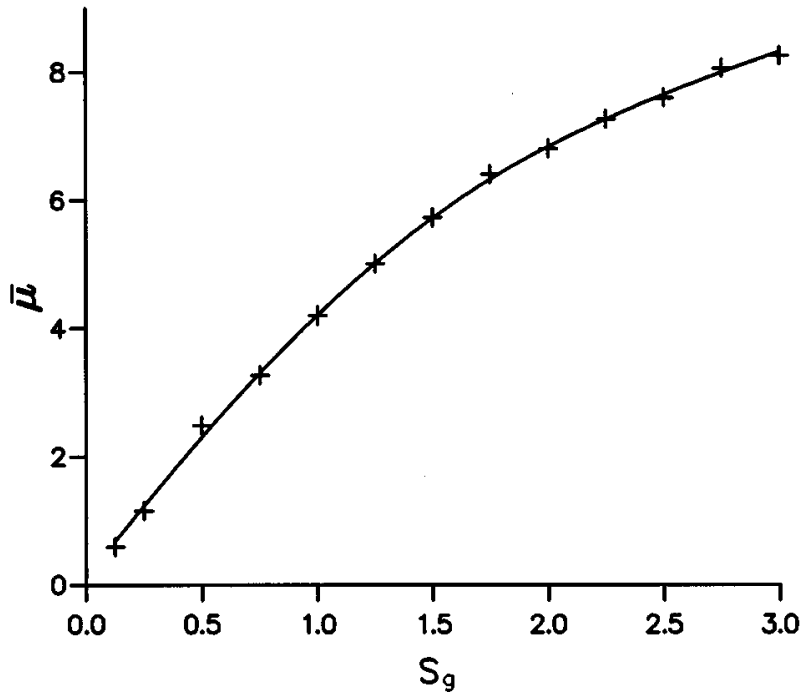

FIG. 9. The variation in the mean number of gravity flips per site per timestep, $\bar{\mu}$, with the gravitational strength parameter, $S_{g}$. The smoothed line through the points can be used to predict the value of $\bar{\mu}$ for any $S_{g}$ between 0.125 and 3.0 .

flips per site per time step, $\mu$, does in general have some dependence on the density. We have already observed that the effect of the density gradient produced across the fluid by the gravitational interactions can be accounted for by scaling the depth by a parameter $\epsilon$ which is assumed to be a function of $S_{g}$. Although some variation in $\epsilon$ has been observed with depth, we have seen that it is adequate to use an average value of $\epsilon$ to describe the whole wave. We extend this assumption here to assume that the density of the whole wave can be described by an average density $\bar{\rho}$ and we can define the mean values of $\mu$ and $\gamma$ to be $\bar{\mu}=\mu(\bar{\rho})$ and $\bar{\gamma}=\gamma(\bar{\rho})$. The mean density $\bar{\rho}$ will be taken to be the average density at which there is non-negligible motion; the density at depths where there is no motion should not be important. To investigate how the mean number of gravity flips $\bar{\mu}$ is related to the implementation strength $S_{g}$ and how it is related to the model parameters, several simulations were set up using a grid size of 2048 by 256 for a number of different $S_{g}$ values in the range $0.125-3.0$. Figure 9 shows the variation in the mean number of particle flips per site per time step, $\bar{\mu}$, with the strength of the gravitational interaction, $S_{g}$. The smoothed line through the points can be used to find $\bar{\mu}$, for any $S_{g}$ in the range $0.125-3.0$. These are effective limits for $S_{g}$, when $R=3$, since below 0.125 the fluid tends to float above the surface due to the long-range interactions pulling the bottom layer of particles upward toward the rest of the body of fluid. Above 3.0 the density gradient is fairly large and the density at the bottom of the wave is large compared to 7, the maximum density. It is worth noting that, as shown in Fig. 10, $\vec{\mu}^{2}$ is approximately linear over this range. Thus $\bar{\mu}$ can be estimated from the equation

$$
\bar{\mu}^{2}=2.52 \times 10^{-5} S_{g}-5.45 \times 10^{-6} ; \quad 0.25<S_{g}<3 .
$$




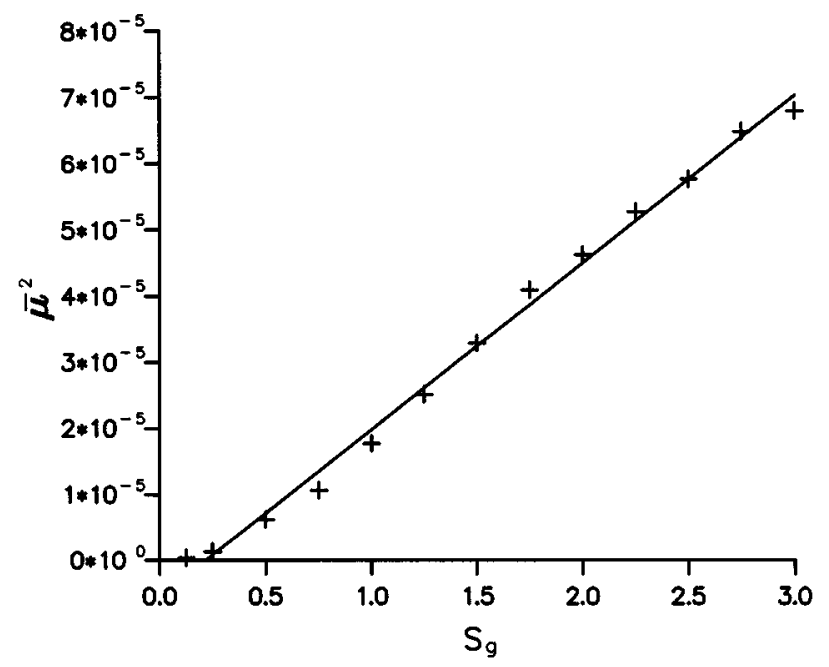

FIG. 10. The approximately linear relationship between $\vec{\mu}^{2}$ and $S_{g}$ and the best-fit straight line through the points.

\section{Relating the mean number of gravity flips to the wave period}

Consider an ensemble of particles each of mass $m$ evolving on $S$ sites for $T$ time steps under gravity. Let the average density of particles be $\bar{\rho}$ and the average number of particle flips per site per unit time be $\bar{\mu}$. The vertical component of the force exerted by gravity on the whole fluid of mass $M$ is given by

$$
F_{y}=M g=S \bar{\rho} m g=\frac{\mathrm{d} P_{y}}{\mathrm{~d} t},
$$

where $P_{y}$ is the vertical component of the particles momentum. The rate of change of vertical momentum due to gravity is given by

$$
\frac{\mathrm{d} P_{y}}{\mathrm{~d} t}=m S \bar{\mu} \delta v_{y},
$$

where $\delta v_{y}$ is the change in the vertical speed of each particle flipped:

$$
\delta v_{y}=\sqrt{3} \bar{\gamma}
$$

Equating Eqs. (31)-(33) we find $g$, the acceleration due to gravity is given by

$$
g=\frac{\sqrt{3} \overline{\mu \gamma}}{\bar{\rho}} .
$$

But from Eq. (17), with the scaling factor $\epsilon$ added, we know that $g$ is given by

$$
g=4 \pi^{2}\left[\tau^{2} k \tanh (k \epsilon h)\right]^{-1} .
$$

Equating Eqs. (34) and (35) we get

$$
\bar{\mu}=B \frac{1}{\tau^{2}},
$$

where

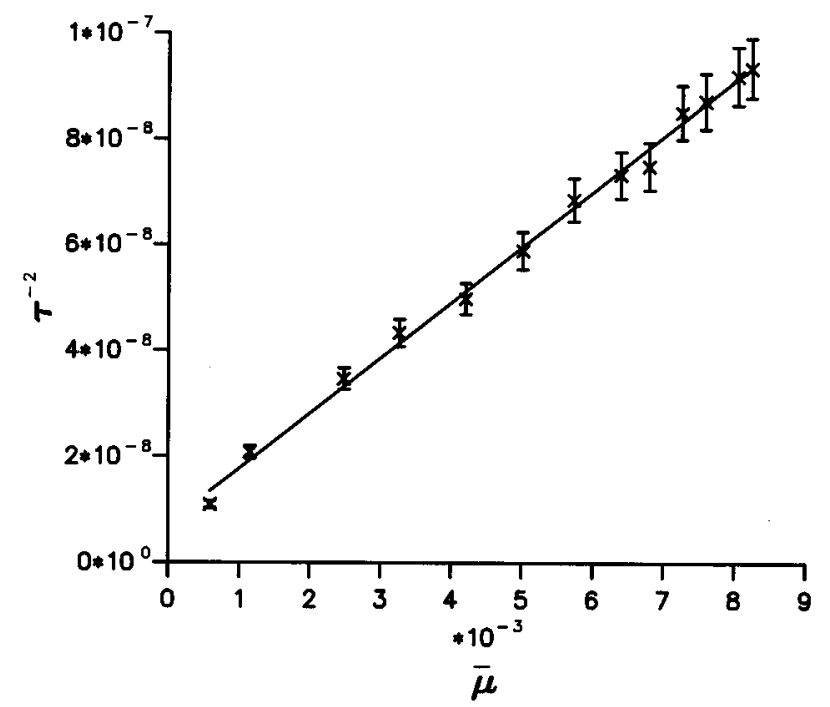

FIG. 11. The variation in the inverse square period $\tau^{-2}$ with the mean number of gravity flips per site per timestep $\bar{\mu}$ and the best-fit straight line through the points.

$$
B=\frac{4 \pi^{2} \bar{\rho}}{\sqrt{3} \bar{\gamma} k \tanh (k \epsilon h)} .
$$

Figure 11 shows the relationship between the inverse squared period $\tau^{-2}$ and the mean number of gravity flips per site per time step. Also shown is the best-fit straight line through the points. The straight line fits through all the points except when $S_{g}=0.125$. We see from Eq. (36) that the gradient of the straight line is $1 / B$, although the straight line fit does not go exactly through the origin. It can be seen from Eq. (37) that $B$ depends on the values of $\epsilon, \bar{\rho}$, and $\bar{\gamma}$. Assuming that $\epsilon$ increases with $S_{g}$, and recalling the values previously found for $\epsilon$ when $S_{g}=0.5$, we can see that the $\tanh (k \epsilon h)$ factor should vary only slowly for $S_{g} \geqslant 0.5$ as it approaches unity. We note that an increase in $\epsilon$ has the effect of increasing the apparent depth causing the motion to take place in a smaller region of the fluid closer to the free surface. Since $\bar{\rho}$ is defined as the average fluid density in which there is motion, that is the average density of the "top" portion of fluid where the wave velocity is non-negligible, an increase in $\epsilon$ will cause the depth at which $\rho=\bar{\rho}$ to move closer to the surface. From Fig. 3 we see that a value of $\bar{\rho}$ $=4.5$ is a good estimate for $S_{g}>0.5$. If $\bar{\rho}$ is approximately independent of $S_{g}$, then $\bar{\gamma}$ should also be approximately constant (as should the viscosity $\nu$ ). Thus we would expect $B\left(S_{g}\right)$ to be approximately constant for larger $S_{g}$ values. We see that this is in fact a good approximation for all $S_{g}$ values in our range. This gives

$$
\tau^{-2} \simeq a \times 10^{-5} \bar{\mu}+9 \times 10^{-9} .
$$

From Eq. (37) we get

$$
\bar{\gamma} \simeq 7.3 \times 10^{-2} \frac{\bar{\rho}}{\tanh (k \epsilon h)} .
$$




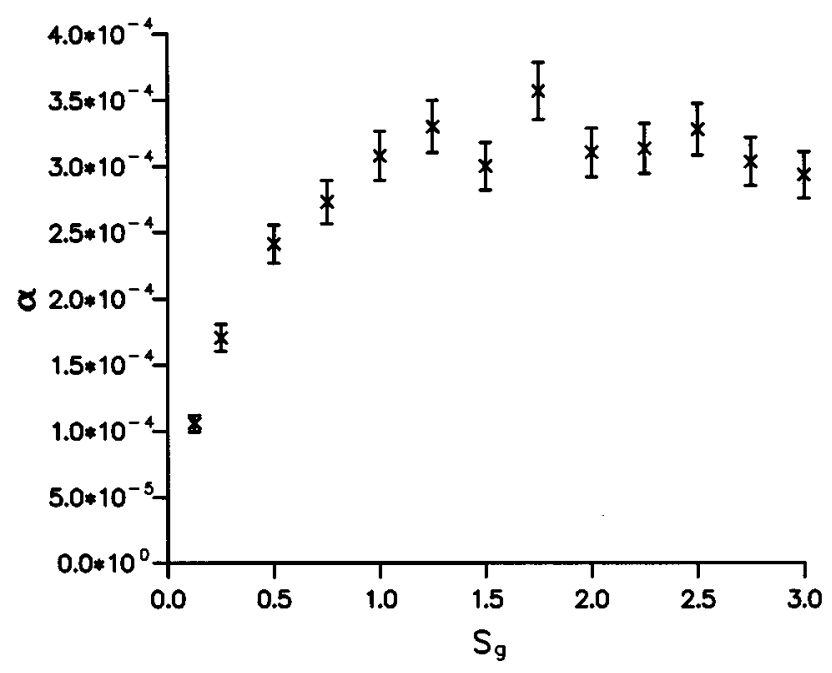

FIG. 12. The variation in the damping constant, $\alpha$, with the strength of the gravitational interaction, $S_{g}$.

\section{The variation of the damping constant with $\boldsymbol{S}_{g}$}

Now consider the dependence of the damping constant $\alpha$ on the gravitational strength, $S_{g}$. As discussed earlier we expect the damping constant, when a free-slip boundary is applied, to be given by

$$
\alpha=\left\{\begin{array}{ll}
2 \nu k^{2} \frac{h}{h^{*}} & \text { when } h<h^{*} \\
2 \nu k^{2} & \text { when } h \geqslant h^{*}
\end{array},\right.
$$

where $h^{*}$ depends on the value of $\epsilon$ through Eq. (23) with $\epsilon$ added:

$$
\epsilon h^{*}=\frac{\lambda}{2} .
$$

Assuming we can write $\epsilon=\epsilon\left(S_{g}\right)$ and that the mean water level $h$ is selected such that $\lambda / 2 \epsilon(3)<h<\lambda / 2 \epsilon(0.125)$, then a plot of $\alpha$ against $S_{g}$ will have two regions, one at lower values of $S_{g}$ where $\alpha$ varies with $S_{g}$ and one at higher values of $S_{g}$ where $\alpha$ is independent of $S_{g}$. We will consider these regions separately. When $h \geqslant h^{*}$ the viscosity $\nu$ is given by

$$
\nu=\frac{\alpha}{2 k^{2}} .
$$

When $h<h^{*}$ we can combine Eqs. (40) and (41) to get

$$
\epsilon=\frac{\pi \alpha}{2 \nu k^{3} h} \text {. }
$$

Figure 12 shows the dependence of the damping constant, $\alpha$, on the gravitational strength parameter, $S_{g}$. The graph can be divided into two section. Initially $\alpha$ is increasing with $S_{g}$, then it remains constant, within the error limits. Due to the noise it is not possible to find the exact value of $S_{g}$ where this changeover takes place, but it is clearly between 0.75 and 1.25 . The later portion, although noisy, suggests $\nu(\bar{\rho})=18 \pm 6 \%$ for $\bar{\rho}=4.5$. Plotting $\alpha$ against $\tanh \left(\sqrt[4]{S_{g}}\right)$, Fig. 13 gives a straight line fit for $S_{g} \leqslant 1.25$

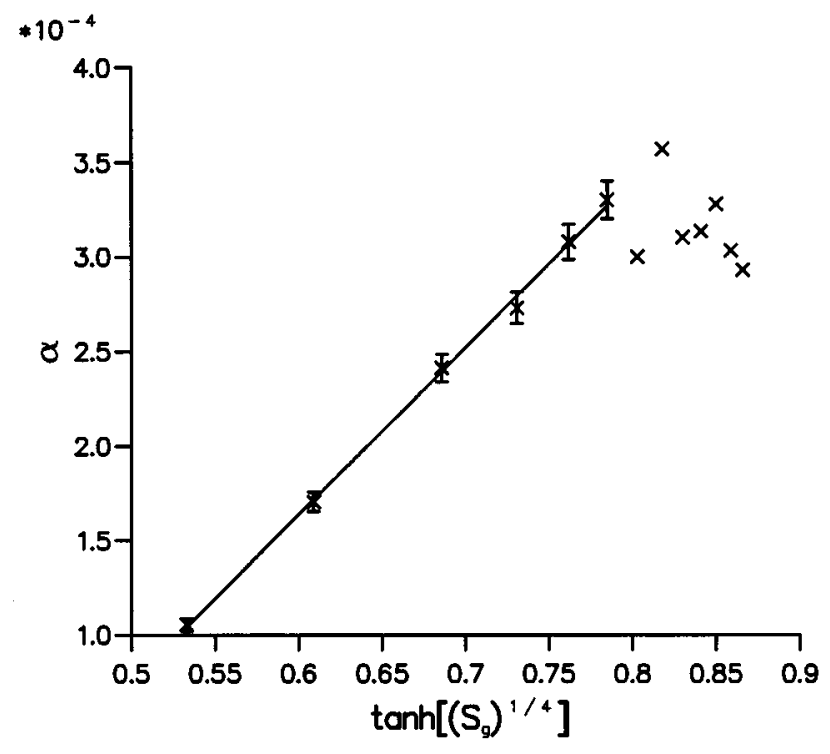

FIG. 13. The variation in $\alpha$ with $\tanh \left[\left(S_{g}\right)^{1 / 4}\right]$ and the best-fit straight line through the points with $S_{g} \leqslant 1.25\left(\tanh \left[\left(S_{g}\right)^{1 / 4}\right] \leqslant 0.78\right)$, the values where there is significant wave motion at all depths.

$\left(\tanh \left(\sqrt[4]{S_{g}}\right) \leqslant 0.78\right)$. The straight line also appears to go through the value for $S_{g}=1.5$ but this may simply be a coincidence. The equation of the best-fit straight line in Fig. 13 is

$$
\alpha=1.06 \times 10^{-3} \tanh \left(\sqrt[4]{S_{g}}\right)-5.08 \times 10^{-4},
$$

which, when combined with Eq. (43), gives

$$
\epsilon=\frac{1}{\nu k^{3} h}\left[9.35 \times 10^{-4} \tanh \left(\sqrt[4]{S_{g}}\right)-4.47 \times 10^{-4} .\right.
$$

This empirical equation, which should in general be true for any value of $h$ and $\lambda$, can be used to calculate $\epsilon$. The equation also relies on the value of the viscosity $\nu$ being known; the value of $\nu$ has been considered earlier.

Thus we find $\epsilon$ to be an increasing function of $S_{g}$ which is approximately unity when there is a negligible density gradient across the depth of the fluid. Physically $\epsilon$ accounts for the density gradient produced across the fluid by the gravitational force. This is significant when $S_{g}>0.125$ (see Fig. 3) but is not considered in standard linear wave theory. When there is a negligible gradient $\epsilon \simeq 1$; however, when the gradient is significant $\epsilon$ can take a value as large as 5 .

\section{Summary of the values found for the model parameters}

The viscosity $\nu$ has been found to be $18 \pm 6 \%$ for $S_{g} \geqslant 1.25,20.8$ and $21.6 \pm 6 \%$ when $S_{g}=0.5$. Given the magnitude of the error in the measurements it is not possible to determine whether the viscosity is remaining constant for all gravitational strengths or whether it is decreasing slightly as the gravitational strength increases. The mean density where motion is observed, $\bar{\rho}$, is approximately constant for all but the smallest value of $S_{g}$ considered so we expect the viscosity to be approximately constant over the range of $S_{g}$ values with value about 21 . 


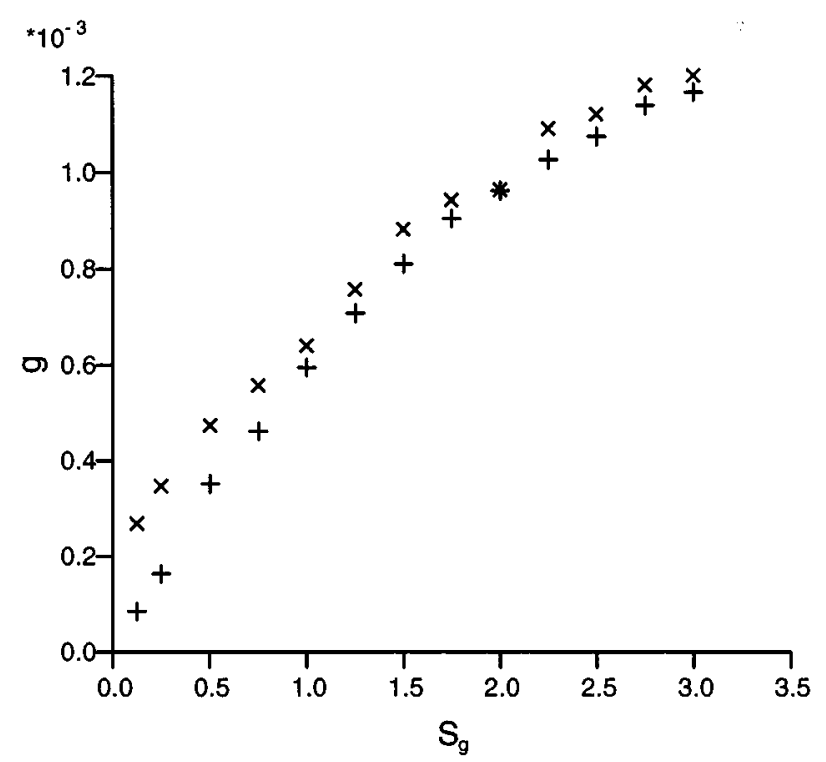

FIG. 14. Graph showing the value of the acceleration due to gravity for different values of $S_{g}$.

The Galilean scaling parameter $\bar{\gamma}$ is also only a function of density and so we again expect that it should be approximately constant for $S_{g} \geqslant 0.25$. From Eq. (37) with $\bar{\rho}=4.5$ and from Fig. 11 we see that $\bar{\gamma} \sim 0.33$ in this range. This is the same as the $\gamma$ value for a FHP-III model with no additional interactions found from Eq. (5) when $\rho=4.3$.

The scaling parameter $\epsilon$ is given by Eq. (45). As before we expect this to be a good approximation for $\epsilon$ when $S_{g} \geqslant 0.25$. We have seen that when $S_{g}=0.125 \epsilon \sim 1$. We do not expect $\epsilon$ to take a value less than unity for any value of $S_{g}$.

The value of the acceleration due to gravity $g$ can be found: (1) from its relation to the wave period $\tau$ through Eq. (17) with $\epsilon$ inserted:

$$
\tau=2 \pi[g k \tanh (k \epsilon h)]^{-1 / 2},
$$

or (2) from Eq. (34) by considering the average number of gravity flips and using the previously found value of $\bar{\gamma}$ and taking $\bar{\rho}=4.5$. The value of $g$ found using both these methods is shown in Fig. 14 where the values calculated by method (1) are represented by $X$ and the values calculated by method (2) are represented by + . These two methods show good agreement for $S_{g} \geqslant 0.75$. For $S_{g} \leqslant 0.5$ the agreement is less good because our assumptions about $\bar{\rho}$ and $\bar{\mu}$ being constant for all $S_{g}$ are less valid. Here the value of $g$ can be found by method (1) since no approximations have been made. The close agreement between the two methods shows that the assumptions made are valid and that the values found for the model parameters are reliable.

\section{Scaling from a lattice system to a real fluid system}

All the results presented here are in terms of lattice units. The unit of length is the separation of the grid sites, the unit of time is the time taken for a particle to move from one site to a neighboring site and the unit of mass is the mass of a particle. To relate the results to real waves we cannot convert the lattice gas units to SI units, but must instead compare waves with the same dimensionless quantities, such as the Reynolds number and the Froude number, as would be done when relating experiments performed in a wavetank to waves in the ocean. As notes above, the dimensionless ratio $a / \lambda \ll 1$ so all the waves are linear. When the dimensionless product $k h$ is such that $\tanh (k h) \simeq 1$ the waves which can be considered as being in deep water. On the other hand, when $\tanh (k h)$ is significantly less than unity, shallow water waves are being considered. The Froude number, which can be taken as $F=c \sqrt{k / g}$, where $c=\omega / k$ is the wave celerity, is simply $F=\sqrt{\tanh (k h)}$ through Eq. (17). The Reynolds number, taken here to be $c \lambda / \nu$, is 21 and 72 for the smallest and largest values of $S_{g}$, respectively. These are relatively low Reynolds numbers so the viscous effects are clearly important. This was observed in the simulation results.

Here there are the additional questions of whether the simulated waves map onto ripples, where surface tension is important, and, given the high viscosity, whether the waves can truly be considered as hydrodynamic. Considering first the influence of the surface tension we consider

$$
k_{m}=\left(\frac{\rho g}{\sigma}\right)^{1 / 2},
$$

where $\sigma$ is the surface tension. When $k \ll k_{m}$ the waves can be considered as purely gravity wave ${ }^{20}$ (as was done here), otherwise the effect of the surface tension must be considered by replacing the dispersion relation, Eq. (17), with ${ }^{20}$

$$
\omega^{2}=\left(g+\rho^{-1} \sigma k^{2}\right) k .
$$

For water waves, surface tension is only important in waves with a wavelength of a few millimeters. In the simulations, where $\sigma$ is of order unity, ${ }^{22} k_{m}$ is significantly smaller than $k$ even for the smallest values of $S_{g}$ so the waves are pure gravity waves. Capillary waves, where there is no gravity and the surface tension is the only restoring force, are considered by Flekkby and Rothman. ${ }^{23}$ When considering a wave in a viscous fluid the parameter

$$
\chi=\frac{\omega^{2}}{g}\left(\frac{\nu}{\omega}\right)^{1 / 2}
$$

determines the affect of viscosity on the wave. ${ }^{24,25}$ If $\chi$ is small then the motion is essentially irrotational except near boundaries where a boundary layer with thickness the order of $(\nu / \omega)^{1 / 2}$ is formed. A boundary layer is not formed at the bottom when free-slip boundary conditions are applied. The values of $\chi$ for the smallest and largest values of $S_{g}$ are 0.39 and 0.29 , respectively. Both are smaller than unity showing that we are in the hydrodynamic regime although, as observed earlier, the viscosity is important.

Another interesting question is whether a liquid-gas wave model which simulates waves between two phases of a single-component fluid, such as at a water-steam interface, can be realistically compared to a two-phase, two-component system such as water and air. At a single-component liquidgas interface there is an exchange of particles, and hence momentum, between the two phases. There can also be an exchange of momentum across a two-component liquid-gas 
interface, for example, the production of ocean waves by the wind, although clearly the mechanisms of the momentum transfer are different, particularly since there is no particle exchange between the phases. During the simulations described here there was no observed deviation from the expected two-component behavior caused by the singlecomponent nature of the model. Any evaporation/ condensation effects were negligible compared to the wave motion in the liquid phase. In the gas phase there were no measurable velocities observed. Thus we conclude that the single-component model is valid for simulating the water-air interface.

\section{CONCLUSION}

We have shown that by applying a gravitational interaction to a separable FHP model we can simulate a dense and a rare fluid separated by a horizontal interface which can be used to model gravity surface waves. When the strength of the gravitational interaction is small the dense fluid had virtually no density gradient across it. When a larger gravitational interaction is used, a density gradient is formed in the dense fluid. We have shown that when a density gradient is produced we can introduce a scaling parameter $\epsilon$ which accounts for the gradient. Waves produced using this model have been seen to behave according to linear wave theory provided the additional scaling parameter is incorporated. Using the model we have found values for the fluid viscosity, the scaling parameter $\epsilon$, the Galilean invariant scaling parameter, $\gamma$, and the acceleration due to gravity for a range of values of the implementation parameter, $S_{g}$.

The model has shown considerable potential for performing surface gravity wave simulations. There are however a few limitations. The fluid viscosity is high, $\nu \sim 20$ compared to the viscosity of an FHP-III model which can be as low as 0.35 . This high viscosity causes the waves to be damped very rapidly even when a large wavelength is used. It is possible to simulate waves which oscillate for up to 10 periods before being completely damped, but this requires a considerable amount of computer time. It is clear from the results presented here that the measurements taken from the simulations are fairly noisy. The noise in density and velocity measurements can be reduced by using larger averaging cells or using ensemble averaging. The noise in measurements of the wave period and the damping constant can be reduced by considering longer simulations and by averaging over a number of results. Any reduction in the level of noise is again at the expense of computer time. The lack of Galilean invariance in the model requires that a scaling parameter $\gamma$ be introduced in order that the fluid obeys the NavierStokes equation. Strictly speaking $\gamma$ is a function of the density $\rho$. We have shown that to a good approximation we can use an average value $\bar{\gamma}$ for the whole fluid despite any density gradient in it. If a Galilean invariant model could be used this approximation would no longer need to be made. These limitations are common to all lattice gas models but they have been overcome for single-component, single-phase models by using a lattice Boltzmann approach ${ }^{26}$ where, rather than considering the individual particles moving on a grid the distribution function, a statistical representation of the fluid, is evolved. The simulation then involves solving the Boltzmann equation ${ }^{27}$ on a regular grid. Details of the development and application of the lattice Boltzmann model are given in Ref. 28. More recently lattice Boltzmann models have been developed which can simulate phase separation and binary fluid mixtures. ${ }^{29-31}$ It is anticipated that the wave modeling ability of the FHP model demonstrated here can be realized in such models.

\section{ACKNOWLEDGMENTS}

This work was supported by the Science and Engineering Research Council. The authors would like to thank P. Stansell for numerous helpful discussions and the Edinburgh Parallel Computing Center (EPCC) for supplying time on the Connection Machine.

${ }^{1}$ R. W. Yeung, "Numerical methods in free-surface flows," Annu. Rev. Fluid Mech. 14, 395 (1982).

${ }^{2}$ J. M. Hyman, "Numerical methods for tracking interfaces," Physica D 12, 396 (1984).

${ }^{3}$ D. H. Peregrine, "Computations of breaking waves,' in Water Wave Kinematics, edited by A. Torum and O. T. Gudmestad (Kluwer, Dordrecht, 1990), pp. 475-490.

${ }^{4} \mathrm{~A}$. Clément, "Coupling of two absorbing boundary conditions for $2 \mathrm{D}$ time-domain simulations of free surface gravity waves," J. Comput. Phys. 126, 139 (1996).

${ }^{5}$ J. Buick, W. Easson, and C. Greated, "Simulation of wave motion using a lattice gas model,' 'Int. J. Numer. Meth. Fluids 22, 313 (1996).

${ }^{6}$ D. H. Rothman, "Cellular-automaton fluids: A model for flow in porous media," Geophysics 53, 509 (1988).

${ }^{7}$ M. Sahimi, "Flow phenomena in rocks: From continuum models to fractals, percolation, cellular automata and simulated annealing," Rev. Mod. Phys. 65, 1393 (1993)

${ }^{8}$ U. Frisch, B. Hasslacher, and Y. Pomeau, "Lattice-gas automata for the Navier-Stokes equation," Phys. Rev. Lett. 56, 1505 (1986).

${ }^{9}$ C. Appert and S. Zaleski, "Lattice gas with a liquid-gas transition," Phys. Rev. Lett. 64, 1 (1990).

${ }^{10}$ D. d' Humieres and P. Lallemand, "Numerical simulations of hydrodynamics with lattice gas automata in two dimensions," Complex Syst. 1, 599 (1987).

${ }^{11}$ D. d' Humières and P. Lallemand, "Flow of a lattice gas between two parallel plates and development of the Poiseuille profile," C. R. Acad. Sci. Paris 302, 983 (1986).

${ }^{12}$ D. d' Humières, P. Lallemand, and G. Serby, "Numerical experiments on lattice gases: Mixtures and Galilean invariance," Complex Syst. 1, 633 (1987).

${ }^{13}$ G. Searby, V Zenlé, and B. Denet, "Lattice gas mixtures and reactive flows," in Discrete Kinetic Theory, Lattice Gas Dynamics and the Foundations of Hydrodynamics (Torino, Italy, September 1988).

${ }^{14}$ P. Clavin, P. Lallemand, Y. Pomeau, and G. Searby, "Simulations of free boundaries in flow systems by lattice-gas models," J. Fluid Mech. 188, 437 (1988).

${ }^{15}$ D. H. Rothman and J. M. Keller, "Immiscible cellular-automaton fluids," J. Stat. Phys. 52, 1119 (1988).

${ }^{16}$ C. Appert, D. Rothman, and S. Zaleski, "A liquid-gas model on a lattice," Physica D 47, 85 (1991).

${ }^{17}$ U. Frisch, D. d' Humières, B. Hasslacher, P. Lallemand, and Y. Pomeau, "Lattice gas hydrodynamics in two and three dimensions," Complex Syst. 1, 649 (1987).

${ }^{18}$ S. Wolfram, "Cellular automaton fluids 1: Basic theory," J. Stat. Phys. 45, 471 (1986).

${ }^{19}$ W. Roberts, “Techniques for Modeling Incompressible Fluid Flow, Ph.D. thesis, The University of Edinburgh, 1991.

${ }^{20}$ J. Lighthill, Waves In Fluids (Cambridge University Press, Cambridge, 1978).

${ }^{21}$ G. D. Crapper, Introduction to Water Waves (Ellis Horwood, Chichester, 1984). 
${ }^{22}$ C. Appert and D. d' Humières, "Density profiles in a diphasic lattice-gas model,' Phys. Rev. E 51, 4335 (1995).

${ }^{23}$ E. G. Flekk by and D. H. Rothman, "Fluctuating hydrodynamic interfaces: Theory and simulation,"' Phys. Rev. E 53, 1622 (1996).

${ }^{24}$ R. D. Dalrymple and P. L. -F. Liu, "Waves over soft muds: A two-layer fluid model,'” J. Phys. Oceanogr. 8, 1121 (1978).

${ }^{25}$ C. C. Mei and L. -F. Liu, "The damping of surface gravity waves in a bounded liquid," J. Fluid Mech. 59, 239 (1973).

${ }^{26}$ Y. H. Qian, D. d' Humières, and P. Lallemand, "Lattice BGK models for Navier-Stokes equation," Europhys. Lett. 17, 479 (1992).

${ }^{27}$ S. Chapman and T. G. Cowling, The Mathematical Theory of Non-
Uniform Gases (Cambridge University Press, Cambridge, 1970).

${ }^{28}$ R. Benzi, S. Succi, and M. Vergassola, "The lattice Boltzmann equation: Theory and application,'” Phys. Rep. 222, 145 (1992).

${ }^{29}$ M. R. Swift, W. R. Osborn, and J. M. Yeomans, "Lattice Boltzmann simulation of nonideal fluids," Phys. Rev. Lett. 75, 830 (1995).

${ }^{30}$ E. Orlandini, M. R. Swift, and J. M. Yeomans, "A lattice Boltzmann model of binary-fluid mixtures," Europhys. Lett. 32, 463 (1995).

${ }^{31}$ M. R. Swift, E. Orlandini, W. R. Osborn, and J. M. Yeomans, "Lattice Boltzmann simulation of liquid-gas and binary fluid systems," Phys. Rev. E 54, 5041 (1996). 